\title{
Face Recognition using SIFT Key with Optimal Features Selection Model
}

\author{
Taqdir \\ Assistant Professor, Computer Science and Engineering \\ Department, \\ GNDU, Regional Campus, Gurdaspur-143521, Punjab, \\ India
}

\author{
Renu Dhir \\ Associate Professor and Head, Computer Science and \\ Engineering Department, \\ NIT, Jalandhar -144001 Punjab, India
}

\begin{abstract}
Facial expression is complex in nature due to legion of variations present. These variations are identified and recorded using feature extraction mechanisms. The researchers have worked towards it and created classifiers for identifying face expression. The classifiers involve Principal component analysis (PCA), Local Polynomial approximation (LPA), Linear binary pattern (LBP), Discrete wavelet transformation (DWT) etc. The proposed work deals with the new classifier using SIFT key with genetic algorithm to identify distinct facial expression. Optimal features of existing algorithms are used within the proposed work. Also comparison of existing techniques such as LBP, PCA and DWT is presented with SIFT key with genetic algorithm. The results show that proposed classifier gives better result in terms of recognition rate.
\end{abstract}

Keywords-Feature Extraction; Classifier; PCA; LPA; LBP; DWT; SIFT key; Genetic algorithm

\section{INTRODUCTION}

Facial recognition system is a biomedical mechanism of identifying various expressions.[1] Facial recognition system is commonly used in security applications but also used heavily in other applications. Facial recognition system involves number of techniques. These techniques are primarily associated with feature extraction. Human face is a house of distinct expression which varies with time continually. Hence efficient classifier is required which generate number of optimal features as quantity to represent entire facial expression residing on human face. Optimal feature selection is difficult with single classifier hence properties of multiple classifiers are collaborated together to achieve optimal classifier.

[2]In this the non-domination based optimization technique has been introduced that recognize the known and unknown faces with a semi-supervised classifier that are based on the different scenarios. The identification is based on proper training sets with actual face images that provide reliable results. In this different datasets like Yale face database, ORL database has been utilized for experimenting and obtaining the robust results. The results are based on TP, $\mathrm{FP}$ and TN classification evaluation. It recognizes the faces by computing LNS of training sets that are considered using the grouping.

FACE RECOGINITION: When features are selected from an image than these feature uses to recognize the faces. In an automated face recognition system, huge diversity is found due to facial appearance during recognition of faces. So many systems are under development now a day that recognises the face based on the appearance. In this system the face image is represented in Eigen faces which consist of vectors of intensities.

There are following tasks which are associated with face recognition system:

$>$ Verification

$>$ Identification

$>$ Watch List

VERIFICATION: The first task of face recognition system is associated with the access applications. The access applications are the one which provide user interaction towards the recognition system. The verification is the process to check the identification of the person that is being access the system using an application. The verification of the persons can be done using two groups:

1) CLIENTS: They are the persons that have access to the system with identity. The percentage rates at which clients are to be rejected are known as False Rejection Rate (FRR).

2) IMPOSTERS: They are the peoples which uses false identity to gain access to the system. With term false identity we mean the identities that are to belonging to the system but known. The imposter gaining access known as False Acceptance Rate (FAR).

IDENTIFICATION: This is done in surveillance application where applications do not need user interaction. It is based on the assumptions that all faces in the Image are known faces. The correct identification percentage can be measured as Correct Identification Rate (CIR) and false identification can be measured using False Identification Rate (FIR).

WATCH LIST: It is the generalized form of identification task in which we will include unknown persons also. To describe the sensitivity of the watch test it includes FAR and FRR along with the identification test reported in CIR and FIR. It describes how often an unknown person tries to access the system. it.

The proposed work has following finding associated with 
- Determining optimal set of features from different training images.

- Comparing Accuracy of different classifiers on training images.

- Comparing different techniques for optimum feature extraction with distinct classifier.

- Comparing performance of different approaches in terms of recognition rate.

The proposed work begins by giving the introduction of classifiers used for face recognition. Next section describes proposed classifier with genetic algorithm. The next section gives the result associated with proposed system. Last section describes conclusion and future work.

\section{FACE RECOGNITION USING PRINCIPAL COMPONENT ANALYSIS}

Face has distinct variations associated with it. Analysing facial expression at distinct time interval is a challenging task. One of the techniques used to analyse facial recognition is with the help of Principal Component Analysis.[3]PCA is a statistical procedure that uses orthogonal transformation to convert possibly correlated values into set of non-correlated linear values known as principal components. It utilizes the set of eigenvalues that are builds from the set of training data sets. From these eigenvalues the training face images have been calculated which are arranged for finding the most variance in image. After this the Euclidean distance from the input face has been calculated for each eigenvalues. This can classified the image into parts based on Euclidean distance. The weighted sum of eigenfaces represented by text face images projected on to the space expanded by eigenfaces. The faces can be identified by these weights.

The following is way through which the correlated values has been calculated:

$$
\begin{gathered}
\mu=\frac{1}{m} \sum_{n=1}^{m} x_{n} \\
C=\frac{1}{m} \sum_{n=1}^{m}\left(x_{n}-\mu\right)\left(x_{n}-\mu\right)^{T}
\end{gathered}
$$

This approach is useful and found application in [4] where it is used to detect emotions. Health of human being is greatly influenced by emotion. Five kinds of emotions are detected in this study. Number of extracted features from the ECG study is reduced by the use of PCA. Also another study using this PCA technique is given in[5] showing emotion detection for movement assisted in wheelchair.

\section{Face Recognition using LBP and LPA}

Image is generally represented either as $2 \mathrm{D}$ or $3 \mathrm{D}$ objects. 2D objects representation is relatively less clear as compared to $3 \mathrm{D}$ objects. Images represented as $3 \mathrm{D}$ objects can be analysed using linear binary pattern. LBP approach used in [6] describes an efficient multimodal face recognition method. This method combines textured as well as depth features extracted from the input image. Linear polynomial approximation and[7] linear binary pattern methods are combined to extract the features and discrete Fourier transformation is used as a transformation tool. The effective face recognition method is achieved with the help of this technique.

\section{FACE RECOGNITION USING LDA}

This approach [8] uses LDA and 2 channel wavelet transformation approach for face recognition. The 2 channel approach is used for factorization of half band polynomial. The analysed system also compares LDA approach with PCA. LDA approach for face recognition is described in this section.

[9]Linear Discriminant analysis is useful to determine combined features that do the separation of the classes. The length and complexity associated with the calculations are reduced using LDA approach. The dimensionality reduction and classification of face recognition is accomplished using least time and space complexity. Distortion within the image is common. This is also accomplished through LDA.

Mathematically, a set of $\mathrm{n}$ dimensional vectors $\mathrm{x}_{\mathrm{i}}, \mathrm{x}_{2},-------$ , $\mathrm{x}_{\mathrm{n}}$ belongs to 1 classes of faces.

$\operatorname{Max} \frac{w^{7} S_{n} w}{w^{7} S_{w} w}$

Where

$\mathrm{S}_{\mathrm{n}}=\sum_{i-1}^{n} n_{i}\left(u^{i}-u_{\text {total }}\right)\left(u^{i}-u_{\text {total }}\right)$

$\mathrm{S}_{\mathrm{w}}=\sum_{i=1}^{n} \sum_{j=1}^{n}\left(x^{i}-u^{i}\right)\left(x^{i}-u^{i}\right)^{i}$

$\mathrm{U}$ is the mean of training images presented to the simulation. $\mathrm{S}_{\mathrm{w}}$ is within the scatter matrix and $\mathrm{S}_{\mathrm{n}}$ is between class scatter matrixes.

\section{FACE RECOGNITION USING DWT}

Face Recognition is critical in identification and verification of a person. This [10]approach using training images and applied with 2D-DWT to obtain LL band features. The LL band features are then normalized so that result lies between 01 and 1.The output obtained is compared against the original image to generate unique features. Gaussian filter [11] is applied in order to remove the noise if any within the image. Further, the feature vectors of many images are combined to form a unique feature vector representing several features. This process also performs compression and enhances recognition rate. The DWT approach is described in this section.

[12]DWT is widely used in numerical and functional analysis. In these areas wavelets are considered to be discretely distributed. DWT has advantage that both location and frequency information is considered. DWT has advantage over Fourier transformation since it has temporal resolution. The concept of wavelet is simple. They are used for multistage analysis process. Description of multistage wavelet is described considering the example as

Example 1

The sequence of wavelets are considered using $n=2^{3}$

$\mathrm{y}=\{1,1,2,3,1,3,2,2\}$ 
Consider vectors $\mathrm{P}$ and $\mathrm{L}$ computed through algorithm for multistage which can be applied as follows

$$
\begin{aligned}
& \text { 1. } P_{I_{-1, J}}=\frac{1}{\sqrt{2}}\left(L_{I, 2 K}-L_{I, 2 K_{-1}}\right) \\
& \text { 2. } L_{I_{-1, J}}=\frac{1}{\sqrt{2}}\left(L_{I, 2 K}+L_{I, 2 K_{-1}}\right) \\
& \text { 3. } I=I-1 \\
& \text { 4. If } \mathrm{i}=0 \text { then stop else move to step } 1
\end{aligned}
$$

The basic ideas behind wavelets are portrayed through the above listed algorithm. The algorithm provides basic understanding of the wavelets or provides compact structure analysis of stored information.

The approaches described above have good recognition rate but it still can further improved. The proposed approach with SIFT key provides better results in terms of accuracy and recognition rate.

\section{PROPOSED TECHNIQUE FOR FACE RECOGNITION}

The proposed scheme combines DWT and LDA method in which result is obtained through decomposition of metrics in four details sub bands. The information obtained is approximation details. The reduced image information is presented to PCA to obtain principal components and reduces dimensionality for storing. The proposed approach is capable of reducing image registration and is highly sensitive to skewing, pin cushioning and vignette that inevitably occurs in images. The proposed work takes the optimal properties of various algorithms along with genetic algorithm to produce optimal rate corresponding to face recognition. The proposed algorithm uses training dataset. The image is selected from training dataset. Median filtering is applied along with clipping operation in order to fetch only face part of the image. Feature extraction module is applied in order to fetch the features from the image. The invariant features are fetched from training set of faces. A feature is a selected image region with an associated descriptor. The descriptor is a special histogram of the image gradients. The gradient at each pixel is regarded as a elementary feature vector which is formed by the pixel location and their gradient orientation. After feature extraction, feature selection is applied. This is accomplished by the use of hybrid approach. In this approach population is initialized. The population consist of features which represent chromosomes. Each feature is fitted with the objective function. The selection process takes place through roulette wheel. In cross over phase chromosome gives rise to new generations. The mutation produces new chromosomes for better generations. In each round a set of similar chromosomes can be generated, these chromosomes may overlap the optimal features for extraction process. The best solution is taken into consideration. Identifying the non-dominated solution and sorting them in Preto front. The non-dominated solutions are copied to the next Pareto solution and the least crowded solutions are also added. The requirements when satisfied algorithm terminates.

The algorithm is listed as follows

Algorithm Face(Training_Set ${ }_{\mathrm{i}}$ )

Input: A set of images describes as training set. Representation as training_set

Test_Image from Training_set $t_{\text {. }}$ Test_Image $=$ Training_set $_{i}$

- Apply Face Acquisition and selection procedure to select particular test image from Training set.

- Apply median filter to reduce noise if any from the image

Test_Image $=$ median2(Test_Image)

- Clipping operation implementation to clip the image to extract only necessary portion of the image.

- Apply Feature extraction based upon descriptor, Feature $=$ Hist(Test_Image)

- Apply Hybridized optimization approach for optimum feature extraction

Repeat the above listed steps until termination criteria is satisfied or optimal result is obtained

\section{RESULTS}

The result produced with the proposed technique is compared with the other approaches. The recognition rate and accuracy is better with this approach as compared to existing approaches. The result in terms of optimal number of features selected is given through training images. 
TABLE I. Optimal Number of FEATURES SElECted Through Proposed APProACH

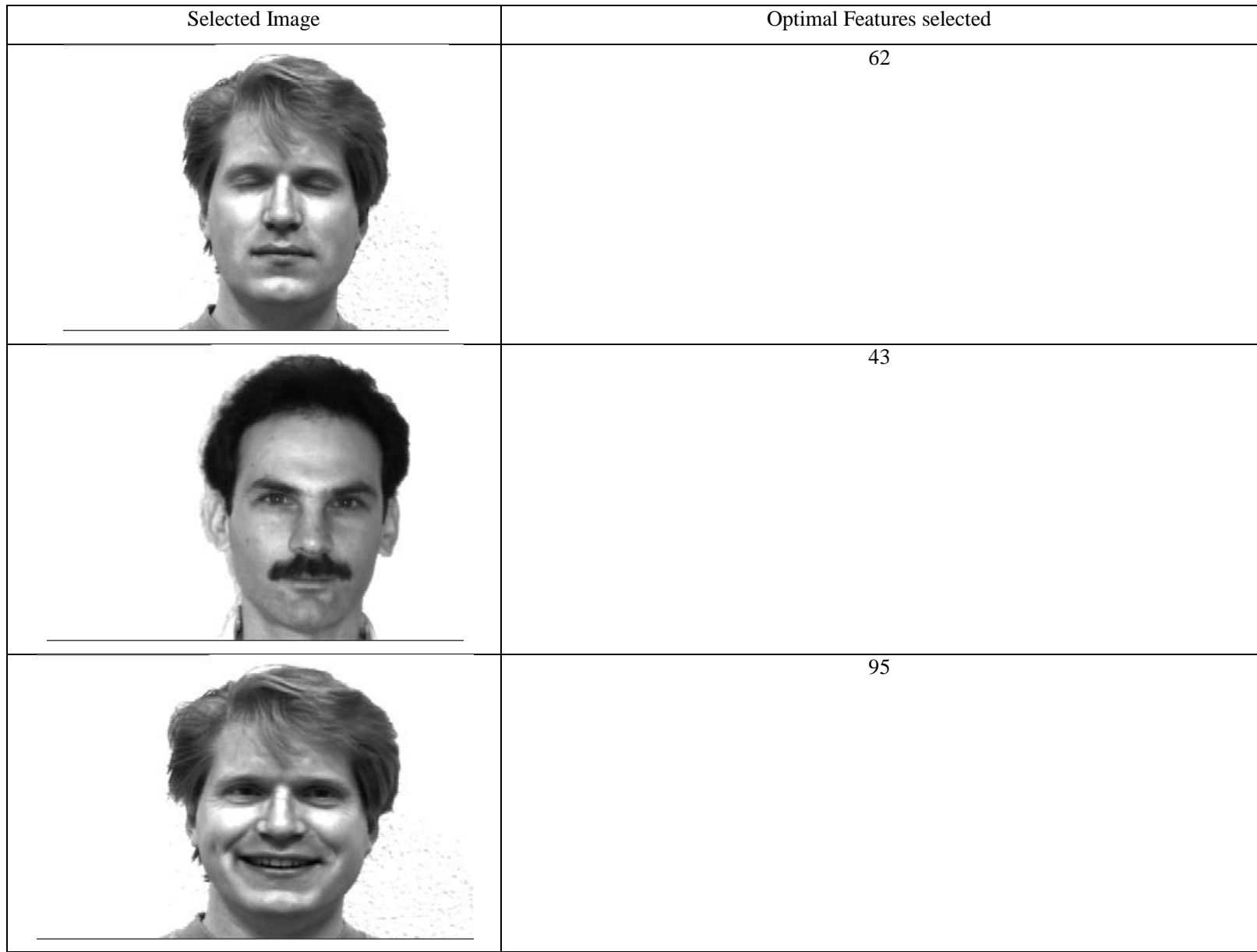

The result generated in terms of accuracy is better as compared to existing approach. The accuracy is calculated in terms of error rate. The error rate is calculated considering the following formula

$$
\begin{gathered}
\text { Error }_{\text {Rate }}=E R F\left(\text { Image }_{i}\right) \\
\text { Accuracy }=100-\text { Error }_{\text {Rate }}
\end{gathered}
$$

The Result generated is listed in the tabular structure.

TABLE II. ACCURACY OF VARIOUS ClASSIFIERS

\begin{tabular}{|l|l|}
\hline Technique with Classifier & Accuracy $(\%)$ \\
\hline PROPOSED & 99.978 \\
\hline CC & 99 \\
\hline DWT & 99 \\
\hline DWT+CC & 99 \\
\hline DWT+PCA & 99 \\
\hline
\end{tabular}




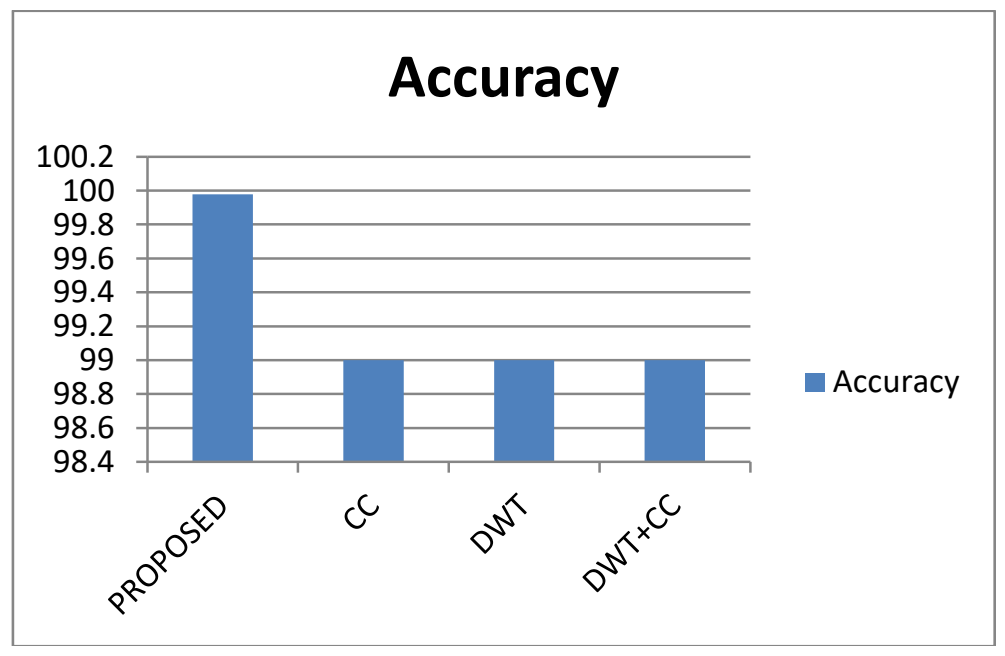

Fig. 1. Accuracy obtained with various classifiers

The optimal feature extracted from various classifiers is shown through tabular structure. The obtained features are best in case of proposed technique.

TABLE III. OBTAINED FEATURES FROM DIFFERENT CLASSIFIERS

\begin{tabular}{|l|l|l|l|l|l|}
\hline Optimum feature selected & Proposed with SIFT & CC & DWT & DWT+CC & DWT+PCA \\
\hline Training Set 1 & 95 & 85 & 93 & 92 & 90 \\
\hline Training Set 2 & 43 & 35 & 42 & 41 & 40 \\
\hline Training Set 3 & 93 & 82 & 90 & 89 & 88 \\
\hline Training Set 4 & 92 & 71 & 89 & 87 & 88 \\
\hline Training Set 5 & 90 & 80 & 88 & 85 & 86 \\
\hline Training Set 6 & 85 & 70 & 83 & 81 & 82 \\
\hline Training Set 7 & 82 & 71 & 78 & 75 & 77 \\
\hline Training Set 8 & 81 & 72 & 89 & 78 & 79 \\
\hline Training Set 9 & 87 & 80 & 88 & 82 & 85 \\
\hline Training Set 10 & 91 & & 86 & 87 \\
\hline
\end{tabular}

For demonstration 10 images from training set are used.

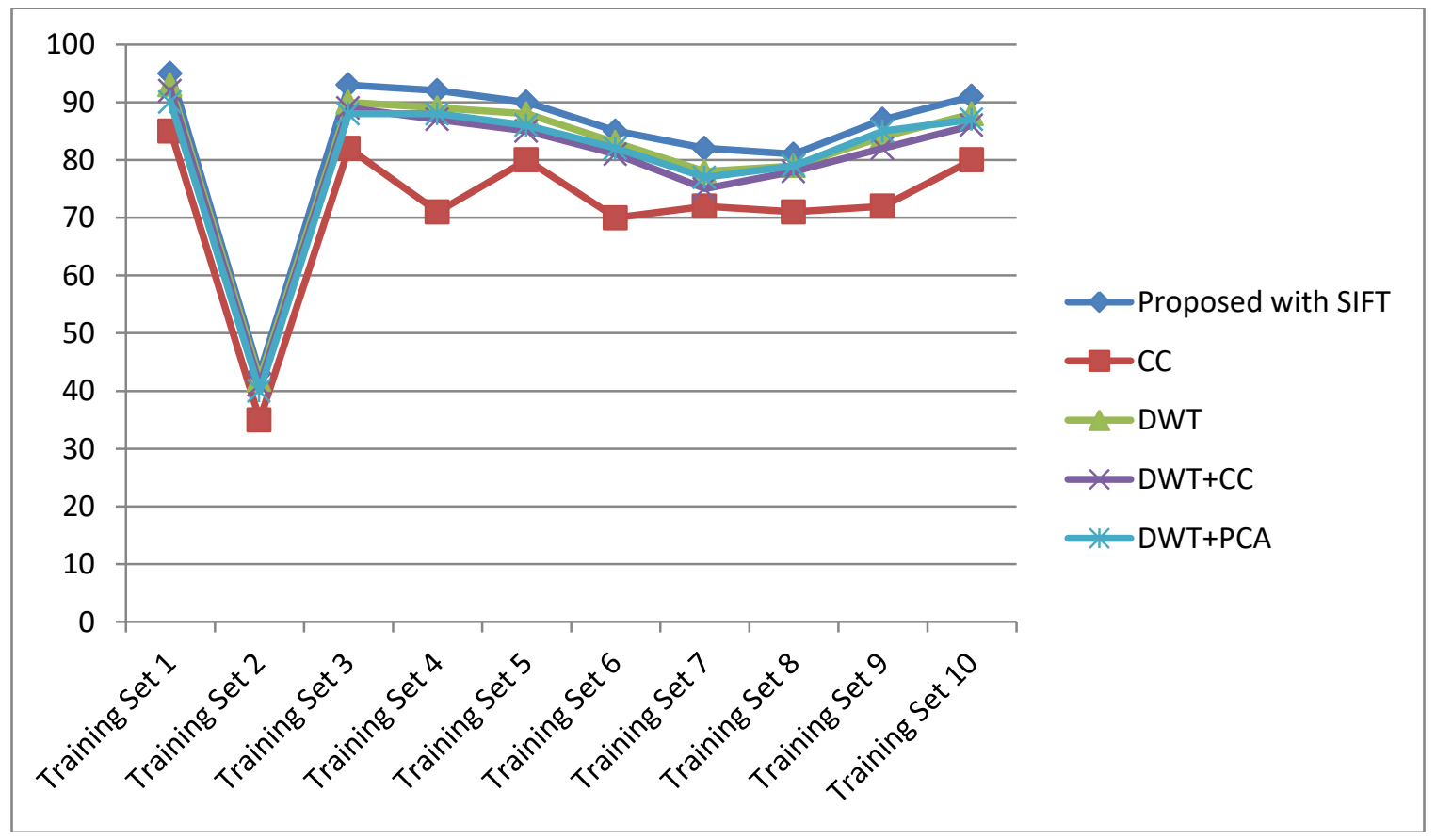

Fig. 2. Obtained features from different classifiers 
(IJACSA) International Journal of Advanced Computer Science and Applications,

Vol. 8, No. 2, 2017

The recognition rate obtained through proposed technique is better as compared to existing approach. This is obtained through simulation. Values are listed through tabular structure as

TABLE IV. RECOGNITION RATE THROUGH VARIOUS ClASSIFIERS

\begin{tabular}{|l|l|l|l|l|l|}
\hline Training set & Proposed with SIFT & CC & DWT & DWT+PCA & DWT+CC \\
\hline Training Set 1 & 98.6087 & 89.7801 & 93.0541 & 93.0401 & 89.8831 \\
\hline Training Set 2 & 96.0223 & 93.2043 & 94.6842 & 94.6662 & 93.1981 \\
\hline Training Set 3 & 72.0652 & 55.9279 & 64.2406 & 64.2447 & 57.6083 \\
\hline Training Set 4 & 92.0232 & 71.3455 & 89.2343 & 87.0873 & 88.6089 \\
\hline Training Set 5 & 90.1143 & 80.9850 & 88.890 & 85.8792 & 86.8769 \\
\hline Training Set 6 & 85.2304 & 70.5744 & 83.3424 & 81.0122 & 82.9034 \\
\hline Training Set 7 & 82.5304 & 72.4763 & 78.2344 & 75.3455 & 77.3423 \\
\hline Training Set 8 & 81.1203 & 71.2342 & 79.2342 & 78.3434 & 79.4555 \\
\hline Training Set 9 & 87.5607 & 72.3443 & 84.2342 & 82.8237 & 85.2332 \\
\hline Training Set 10 & 80.3434 & 88.3453 & 86.7878 & 87.0452 \\
\hline
\end{tabular}

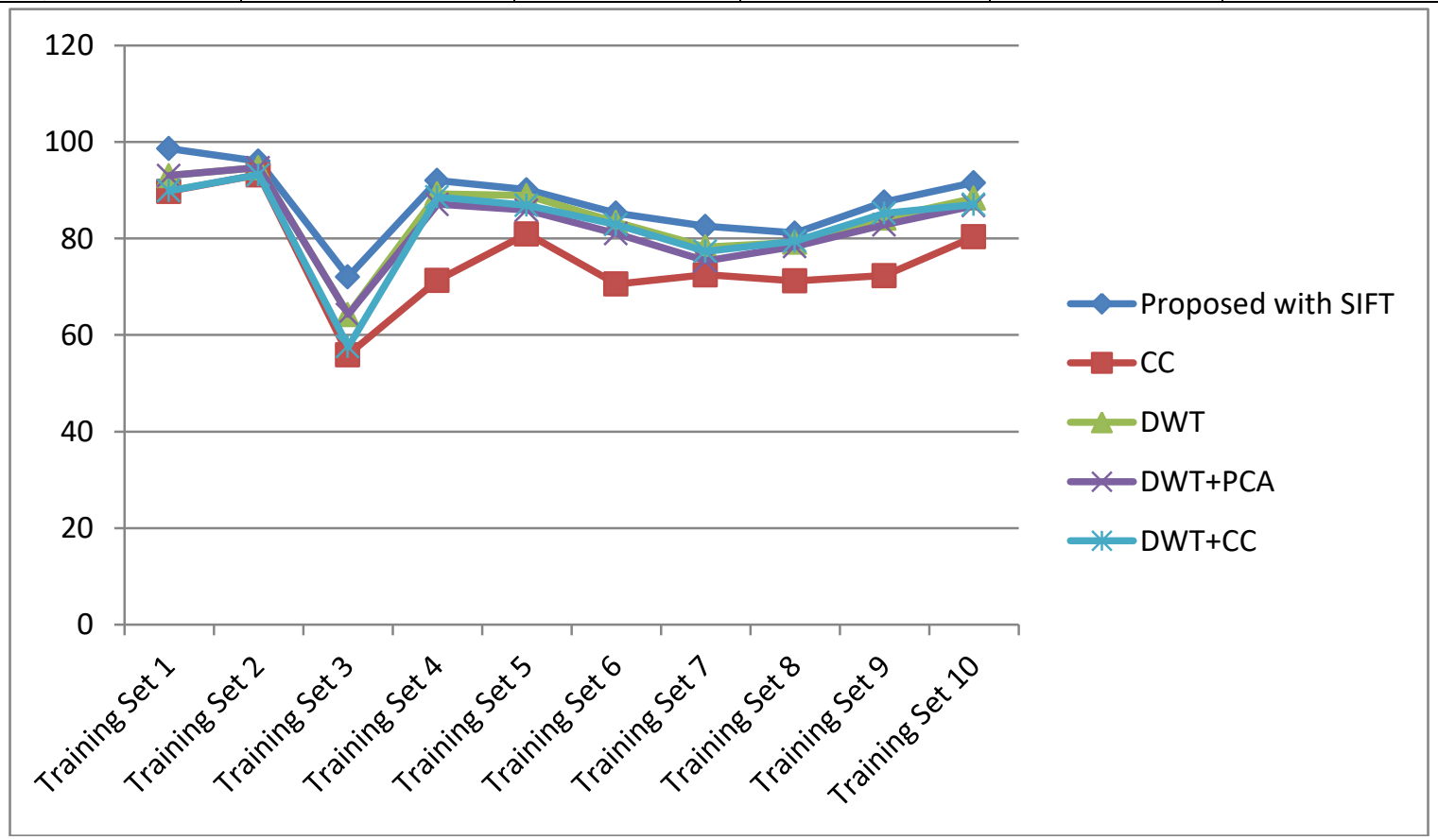

Fig. 3. Recognition rate through various classifiers

Results shows that proposed technique is better in terms of number of different characteristics. These characteristics include recognition rate, accuracy, optimal number of features and obtained features through classifiers.

\section{CONCLUSION AND FUTURE SCOPE}

Automatic selection of features is greatest advantages of the proposed technique. The feature selected is fed into the system for optimality. Since the process is iterated hence result obtained is filtered with iteration. The results are compared against different classifiers to prove the validity of the approach. The feature selection is based on objective function value. Better convergence in terms of recognition rate is presented through proposed technique. The result is better in terms of recognition rate, accuracy and number of optimal features generated.

The proposed technique utilizes hybrid approach to obtain better convergence in terms of recognition rate. The genetic algorithm which is used is situation dependent. By saying so we mean it may converge better for some of training sets better as compared to other training sets. Unguided mutation is also problem with the proposed genetic approach. In future hybrid approach of Ant colony and Honey bee can be used for achieving enhanced optimality.

\section{REFERENCES}

[1] "What is facial recognition? - Definition from WhatIs.com." [Online]. Available: http://whatis.techtarget.com/definition/facial-recognition. [Accessed: 29-Dec-2016].

[2] T. kaur and R. dhir, C. Science," Hexagonal Descriptor Particle Swarm Optimization with Knowledge-Crowding for Face Recognition",SERSC, EI compendex, IJSIP vol. 9, pp. 253-264, 2016.

[3] T. Pecchia, A. Gagliardo, C. Filaninno, P. Ioalè, and G. Vallortigara, "Metadata of the chapter that will be visualized in SpringerLink AdultBorn Neurons in the Olfactory," Behav. Lateralization Vertebr., 2012.

[4] P. Adibi and S. Ahmadkhani, "Face recognition using supervised probabilistic principal component analysis mixture model in dimensionality reduction without loss framework," IET Comput. Vis., vol. 10, no. 3, pp. 193-201, Apr. 2016.

[5] H.-W. Guo, Y.-S. Huang, C.-H. Lin, J.-C. Chien, K. Haraikawa, and J.S. Shieh, "Heart Rate Variability Signal Features for Emotion Recognition by Using Principal Component Analysis and Support Vectors Machine," in 2016 IEEE 16th International Conference on Bioinformatics and Bioengineering (BIBE), 2016, pp. 274-277. 
[6] M. C. Sobia, V. Brindha, and A. Abudhahir, "Facial expression recognition using PCA based interface for wheelchair," in 2014 International Conference on Electronics and Communication Systems (ICECS), 2014, pp. 1-6.

[7] Naveen S., S. S. Nair, and R. S. Moni, "3D face recognition using optimised directional faces and fourier transform," in 2015 International Conference on Advances in Computing, Communications and Informatics (ICACCI), 2015, pp. 1856-1861.

[8] J. Ren, X. Jiang, and J. Yuan, "LBP-Structure Optimization With Symmetry and Uniformity Regularizations for Scene Classification," IEEE Signal Process. Lett., vol. 24, no. 1, pp. 37-41, Jan. 2017.

[9] M. A. Muqeet and R. S. Holambe, "Face recognition using LDA based generalized half band polynomial wavelet filter bank," in 2016 International Conference on Electrical, Electronics, and Optimization Techniques (ICEEOT), 2016, pp. 4649-4653.

[10] D. Marculescu, N. H. Zamora, P. Stanley-marbell, and R. Marculescu, "Fault-Tolerant Techniques for Ambient Intelligent Distributed Systems-," pp. 348-355, 2003.
[11] G. V Sagar, S. Y. Barker, K. B. Raja, K. S. Babu, and Venugopal K R, "Convolution based Face Recognition using DWT and feature vector compression," in 2015 Third International Conference on Image Information Processing (ICIIP), 2015, pp. 444-449.

[12] Singh, "Performance Comparison of Various Image Denoising Filters Under Spatial Domain," vol. 96, no. 19, pp. 21-30, 2014.

[13] Xue B. Zhang M., Browne W. N., "Particle Swarm Optimization for Feature Selection in classification: A Multi-Objective Approach", IEEE Transactions on Cybernetics, pp. 1-16, 2012

[14] T. Kaur and R. Dhir, "Feature Optimility Based Semi-Supervised Face Recognition Technique" ,chapter in Advs in Intelligent Syst., Computing, Vol. 515, proceeding of the 5th international conference on frontiers in intelligent computing.

[15] T. Kaur and R. Dhir, "A Non-domination Pareto-based Scale-Invariant Approach for Face Recognition,” Eur. J. Eng. Res. Sci., vol. 1, no. 2, pp. 6-13, 2016. 